\title{
Electrical Disturbances and the Nature of Electrical Enerǵy*
}

\section{By Charles P. Steinmetz}

To all of us who are interested in the use of electric energy the nature and characteristics of electric energy are of importance; as on their understanding depends our success in the economic use of this energy, and our ability of guarding against the difficulties, troubles and dangers which it may threaten when out of control.

Electric energy is industrially used as direct current and as alternating current, that is, as steady flow and as wave motion, usually of 2.5 or 60 cycles. Electric disturbances are of various character, and, where they are periodic

It can not well be doubted that electric disturbances in our systems are increasing in number. The reason therefor is found in the increasing size and energy of modern electric systems. Just as in a pail of water even a gale will not cause an appreciable disturbance, or, as a small pond is usually quiet while the ocean is never at rest, but continually traversed by undulations from small ripples to big waves, so in a small isolated plant high voltage disturbances are practically unknown; are rare in smaller central stations; while in the huge modern systems waves continuously traverse the circuits, from minute high frequency ripples of negligible energy to occasional high power surges of destructive energy
energy.

The nature and form of disturbances met in electric systems are as variegated as those of any other form of energy. Single electric waves or impulses may appear as magnetic discharges, analogous to the snap of a whip in acousties. Oscillations appear as waves which start suddenly and gradually die out, like the waves produced by throwing a stone in water; such are the disturbances caused by switching, synchronizing, ete. Then there are traveling waves, analogous to the ocean Then there are traveling waves, analogous to the ocean
waves, of various size and wave length; such for inwaves, of various size and wave length; such for in-
stance as the disturbances caused by arcing grounds, by lightning, etc. Standing waves or stationary oseillations, like those of a tuning fork or violin string may appear; and occasionally also, the most dangerous of all disturbances, cumulative oscillations, like the resonance of a tuning fork, namely, oscillations which gradually build up, increase in intensity until they finally limit themselves and become stationary, or die down again, or increase until something happens. Such for instance are the hunting of synchronous machines, for instance are the hunting of synchronous
certain internal transformer oscillations, etc

certain internal transformer oscillations, etc.
Disturbances may affect the system by their quantity, or by their intensity. Electric power can he resolve into the product of two terms, quantity (or current) and intensity (or voltage), just as most other forms of energy are resolved into the product of two terms. Hydraulic energy is quantity of water times head or pressure; heat energy is entropy times temperature pressure; heat energy is entropy times temperature,
etc. Instances of current disturbances are the moetc. Instances of current disturbances are the mo-
mentary short circuit currents of alternators, the very high frequency currents of arcing grounds, etc. Voltag disturbances appear wherever an electric wave breal at a barrier in a circuit, as at a reactance, or in the end turns of a transformer.

A wave in the water, as a big ocean wave, may cause damage by its bulk, by overturning a structure. So a current wave may cause damage by its volume: the momentary short circuit current of an alternator may tear the windings to pieces, twist off the engine shaft tear the windings to pieces, twist off the engine shaft,
etc. Again, waves in the water too small of themselves etc. Again, waves in the water too small of themselves
to do any harm, may still do harm by the continuou pounding-by undermining and washing away the shore. In such manner a continuous oscillation-a electric impulse would not have sufficient energy to do damage, but when they follow each other successively, in thousands and millions, as coming from an arcin ground, then finally they cause destruction. Again ground, then finally they cause destruction. Again,
the damage may be done by the pressure or voltage. Just like an ocean wave, not high enough in itself to overtop the shore, when stopped at the beach, whe breaking in the surf, throws the water up to height that are much greater than the height of the wave, so in the same manner a voltage impulse in an electric distribution system, when it breaks at the entrance to another circuit, at a reactance, or the end connection of the transformer or generator, or the series coil of a potential regulator, may pile up high voltage and rise to values far beyond those which the wave has in its free path in the cable or the line; and there, at
the point where it breaks, where the wave is abruptly *.An address to the Association of Edison Illuminating Com
panies at their convention in spring Lake Beach, N. Published in the General Electric Review and reprinted in the

stopped by reactance, the voltage may rise to destructive

Disturbances may enter the electric system from the outside, as by lightning; or they may originate in the system, as by switching, synchronizing, etc. or again, they may originate in the circuit by outside interference, as by an arcing ground, a spark discharge to an isolated conductor, etc.

A characteristic of most of these disturbances (which usually are comprised by the name of transients) i that they easily pass from circuit to circuit across space by magnetic or static induction, but frequently do tance. The cause of this is found in their nature, particularly the frequency.

When an electric current passes through a circuit there is in the space surrounding the conductor which carries the current an electric field; lines of magnetic force surround the conductor, and lines of electrostatic or dielectric force radiate from the conductor. In a direct current circuit, if the current is continuous, the field is constant; there is a condition of stress in the space surrounding the conductor, which represents stored energy, magnetic energy and dielectric energy, just as a compressed spring or a moving mass represents stored energy. In an alternating urrent circuit, the electric field also alternates; that is, with every half wave of current and of voltage, the magnetic and the dielectric field start at the conductor and run out from the conductor into space with the velocity of light, or 188,000 miles per second. Where this alternating field of the conductor, this electric wave, impinges on another conductor, a voltage and a current are induced therein. The induction is proportional to the intensity of the field (the current and voltage in the conductor which produce the field) and to the frequency. Thus, where the frequency is extremely high, intense induction occurs; that is, considerable energy is transferred from the conductor which produces the electric wave (the primary or sending conductor) to any conductor on which the wave impinges (the secondary or receiving conductor). The result is, that a large part of the energy of the primary conductor passes inductively across space primary conductor passes inductively across space
into secondary conductors, and the energy decreases rapidly along the primary conductor. In other words, such a high frequeney current does not pass for long distances along a conductor, but rapidly transfers it energy by induction to adjacent conductors. This higher induction, resulting from the higher frequency, is the explanation of the apparent difference in the propagation of high frequency disturbances from the propagation of the low frequency power of our alterpropagation of the low requency power of our alter-
nating current systems: the higher the frequencv, the more preponderant become the inductive effects, which transfer energy from circuit to circuit across space, and therefore the more rapidly the energy decreases and the current dies out along the circuit, that is, the more local is the phenomenon.

The flow of electric power thus comprises phenomena inside of the conductor, viz., the dissipation of electric energy by the resistance of the conductor through its conversion into heat and phenomena in the space outside of the conductor - the electric field-which, in a continuous current circuit, is a condition of steady magnetic and dielectric stress, and in an alternating current circuit is alternating, that is, an electric wave issuing from the conductor and traveling through space with the velocity of light. In electric power of the conductor are of main importance, and the electric field of the conductor is usually observed only incidentally, when it gives trouble by induction in incidentally, when it gives trouble by induction in
telephone circuits, or when it reaches such high intensities as to puncture insulation, cause mechanical motion, ete. Inversely, in the use of electric power for wireless telegraphy and telephony, it is only the electric field of the conductor, the electric wave, which is of importance in transmitting the message; the phenomen in the conductor

\section{re not used.}

The electric waves of commercial alternating current circuits usually have the frequencies of 25 and 60 cycles. With a velocity of propagation of 188,000 miles per
second, 25 waves per second give a wave length of $\frac{188,000}{25}$

The distance to which the

field of a transmission line extends is, therefore, only an insignificant part of the wave length, and the phase difference within the field of the transmission line thus is inappreciable. With the alternating fields of transof the field is therefoct of the velocity of propagation Not so with the aiternating field of a wireless telegraph station. Using frequencies from one hundred thousand to a million cycles, the wave length is from $\frac{188,000}{100,000}=1.8$ miles to $\frac{188,000}{1,000,000}=0.188$ miles, or about 1,000 feet. With a wave length of from 1,000 feet to 2 miles, the electric wave extends over hundreds of cycles within the operative radius of a wireless telegraph station, which may be hundreds or even thousands of miles. It is appreciable also in long distance telephone lines. The average frequency of the sound waves500 eycles-gives a wave length of 376 miles, and a 1,000-mile telephone line thus comprises over $2 \frac{1}{2}$ waves. That is, at the moment when one-half eycle of telephone current arrives in Chicago from New York, five succeeding half waves have already left the New York terminal and are on the way.

Abnormal electric waves in industrial electric power circuits vary from a few eycles per second (in the stationary oscillations of compound electric circuits) up to thousands, hundreds of thousands and millions of eycles per second. At frequencies of many thousand cycles per second, the ordinary measuring instruments, the oscillograph, etc., fail to record the wave; but such very high frequency waves can still be observed and measured through their inductive effects by bringing a conductor near them: the electric wave, impinging on this exploring conductor ("resonator" or "receiving antenna") then induces a current in it, and this is observed by a sufficiently delicate apparatus. In this manner, the telephone disturbances caused by alternating electric railway circuits have been studied by exploring antennae. A very intense wave, at short distance from its origin, may be observed by the spark across a small gap in the exploring antenna. Inversely, at hundreds of miles distance from the wireless sending station, the extremely weak wave is still observed in the receiving antenna by a change of the surface tension of a platinum hair wire dipped into an electrolyte, the change in resistance of which operates a relay. By the exploring antenna, electric waves have been studied and observed up to frequencies of hundreds of millions of eycles per second-so-called "Hertzian waves,"-as they occur in industrial circuits between the end eylinders of high voltage multi-gap lightning arresters. There, they are the cause of the high sensitiveness of the arrester for high frequency disturbances. We have to realize though, that light and radiant heat, the Hertzian waves, the waves of the wireless telegraphy station, the alternating fields of our transmission and distribution circuits, are one and the same phenomenon-electric waves traveling through space with the same velocity $(188,000$ miles per second) and exhibiting the same characteristics, but differing merely by their frequencies. This does not mean that electricity and light are the same, but that light is an extremely high frequency electric wave, an extremely rapid alternating electric field, while the electric field of the direct current is a steady stress in space.

From our knowledge of the identity of the alternating electric field and the wave of light radiation, we can derive a number of interesting relations between electric phenomena and the phenomena of light. To mention only one: the secondary current is repelled by the alternating magnetic field which induces it, that is, by the electric wave impinging upon it. This fact is made use of in the constant current transformer for constant current regulation. Applying the same phenomenon to the extremely high frequency light waves, means that the body which intercepts the light wave is repelled by the wave-the radiation pressure. Thus at extremely high frequencies the radiation pressure is the analogous phenomenon to the repulsion between primary and secondary circuits in our industrial eircuits.

So far we have made no hypothesis, but merely recorded the facts: we can measure the waves and their frequencies, their velocity of propagation and other characteristics, and show their identity. We may now speculate on the nature of the electric wave, on the mechanism of its propagation, etc.; but must then realize, that as soon as we leave the facts and indulge in speculation, we submit to uncertainty, which every hypothesis has, no matter how well founded.

The velocity of propagation of the electric wave is incredible, but it is a finite velocity, and after the elapses before it is observed by the receiving antenna. 
The energy sent out by the oscillator, the electric circuit, the sending antenna, is thus received by the propagation of the electric wave implies that the energ during its motion from the starting point to the point observed must reside for some time in intervening space. This means that there must be something in the space which carries the energy; a carrier of the energy of radiation, of light. That carrier we explain by the hypothesis of the luminiferous ether. We assume that the ether permeates all space, is of extreme tenuit and fineness, and is the carrier of the electric wave The question arises: Is the ether a mere hypothesis or is it real? Is it a form of matter or not? We may speculate on that, but may come to one conclusion
or to the opposite conclusion, according to our definior to the opposite conclusion, according to our defin-
tion of what matter is. After all, it is really not a question of what matter is. After all, it is really not a ques-
tion of speculation, but a question of definition-of tion of speculation, but a
what you define as matter.

We always speak of the phenomena of nature within the conception of energy and of matter. Energy we can perceive by our senses. All we know of nature all that our senses give us as information, is the effect of energy--energy which reaches our body through the eyes, through the ear, through the sense of touch, and if I were to make a definition of energy it would be "that thing which reacts on, and is perceived by, or "that thing which reacts on, and is perceived by, or the most consistent definition of energy.

Now, what is matter? We cannot see or get any knowledge of matter. If we see a thing, we do not see the matter, but we see the radiating energy from it which comes to us. We feel the mechanical energy of its momentum, but the matter we cannot perceive. All the conception of matter is as the carrier of energy but if you define matter as the carrier of energy, then but if you define matter as the carrier of energy, then
the ether which carries radiating energy-carries the the ether which carries radiating energy-carries th
energy of the electric wave-is just as much matte as the bullet which carries the mechanical energy that was supplied to it in the gun.

The question then arises: What are the propertie of this ether, which is the carrier of the electric wave? The velocity of propagation of a wave in a medium depends on its density and elasticity. The velocity
of propagation of the electric wave through the ether of propagation of the electric wave through the ether
is nearly 200,000 miles per second, while the velocity is nearly 200,000 miles per second, while the velocity
of sound waves through the air is about 1,000 feet per second, or the electric wave moves a million time faster than the sound wave. This means that the ether must be of a density inconceivably lower than that of air, though we speak of the air as being of low density, and realize this when trying to navigate it. Furthermore, through the ether all cosmic motion takes place: our earth rushes through it at high velocity, takes place: our earth rushes through it at high velocity
and still there is no appreciable friction. That means and still there is no appreciable friction. That means
that the density of the ether must be so enormously that the density of the ether must be so enormously
low that even at very high velocity the frictional resistance is inappreciable.

We might then consider the ether as a gas of incon eivably low density.

However, the light wave or electric wave is a transverse vibration; that is, the oscillating ether particles oscillate at right angles to the direction in which the oscillate at right angles to the direction in which the
ray of light travels, and therefore in their oscillation ray of light travels, and therefore in their oscillation
come neither nearer nor recede further from the ether come neither nearer nor recede further from the ether
particles in front or behind in the direction of the beam of radiation. The oscillation cannot be transferred from ether particle to ether particle in the direction of the beam, by approach or recession of the ether particles, and the transfer of oscillation in the direction of the beam thus can occur only by some thing, some force, which holds the ether particles together, so that a side motion of one causes a corresponding side motion motion of one causes a corresponding side motion
of the particle ahead, without approach. That is, of the particle ahead, without approach. That is, the ether particles can not be free as in a gas, but must
be held together with some rigidity. In other words, the existence of transverse vibrations precludes that the ether is a gas, and requires it to be a rigid body, a solid: transverse oscillations can occur only in solids, but are inconceivable in fluids. From the nature of the wave motion of light, we thus would have to conclude that the ether, through which the earth and al bodies rush with high velocity, and without appreciable bodies rush with high velocity, and without appreciable
friction, is a solid. This is physically impossible, and friction, is a solid. This is physically impossible, and
here we find a very common physiological phenomenon if we attempt to carry any speculation or theory to it final and ultimate conclusion, we reach contradiction This probably is not the result of the nature of the phenomena, but is in the nature of our minds, which are finite and limited, and therefore fail when attempting to reason into the infinite.

A speculative hypothesis on the nature of electrical phenomena has in the last years been developed in the ionic theory. Its starting point is the study of the phenomena of conduction, more particularly the conduction of gases and vapors. In this, we must not merely consider typical cases, but cover the entire
field of conductors. On first sight, it appears easy to

* Compare w. Ostwald: "Energy is that which can be distinguished in time and divide all electric conductors in two classes: metallic conductors or conductors of the first class, in which the resistance slightly increases with increase of temperasecond class, in which the resistance slightly decrease with the temperature. Further investigation shows, however, that there are numerous conductors which however, that there are numerous conductors which
do not belong in either class, such as gases, vapors, etc., and that there are all transition stages between the different conductors represented, so that we can not speak of classes any more, but merely of types. Thus there are solid conductors, such as metallic oxide (for instance magnetite) and elements and their alloys, gradually change from metallic conductors of positive temperature co-efficient to conductors of metallic temperature co-efficient to conductors of metallic
character, but with negative temperature co-efficient; character, but with negative temperature co-efficient;
and which at still other temperatures have such high negative temperature co-efficients that the voltage decreases with increase of current, thereby having the same characteristics as are conductors; while at stil higher temperatures they become electrolytic conductors. Such "pyroelectrolytie" conductors, to which the Nernst lamp glower belongs, are interesting because of the change of type of their conduction. Equally, if not more interesting, are gases and vapors as conductors, such as the arc, the Geissler tube, the static spark, etc. There seem to exist two classes of gas or vapor conduction: to the one belong the arcs, while
to the other belong the Geissler tube and the electrostatic spark. Again, on first sight, it appears difficul to realize that the silent faintly luminous Geissler tube discharge, and the brilliant and noisy electrostatic spark, are one and the same phenomenon. However, the one changes gradually and without dividing line into the other by a change of gas pressure, and the into the other by a change of gas pressure, and the
difference in the gas pressure. Furthermore, the usual noise and brilliancy of the static spark at atmospheric pressure is largely the result of the circuit condition under which it is produced: the passage of the spark closes the circuit and thereby starts a momentary more or less unlimited flow of electric energy. If, however, this short circuiting effect of the spark is eliminated, as for instance by interposing between the spark terminals a glass plate which is not punctured, the electrostatic sparks appear as thin colored moderately luminous discharges which pass with moderate noise, the apparent difference from the Geissler discharge being then far less. With decreasing gas pressure, the electrostatic spark becomes less noisy, less brilliant,
longer and thicker, and finally changes to the noiseless steady stream of the Geissler discharge, which traverse the space between positive and negative terminal with a glow, its color depending on the nature of the gas: for example, the glow is pink with air, orange-yellow for example, the glow is pink with air, orange-yellow
with nitrogen, green with mercury vapor, ete. Going still to higher and higher vacua, the conductor which passes the current between the positive and negative terminal of the vacuum tube finally changes again and becomes a green discharge, which issues from the negative terminal in straight lines, like a beam of light, It may not reach or come anywhere near the positive terminal, and if the positive terminal is located back of the negative terminal, the eathode ray, issuing from of the negative terminal, the cathode ray, issuing from
the latter, will really proceed away from the positive terminal.

Now, this form of electric conduction (and to a considerable extent the conduction of the Geissler tube at lower vacuum) looks very much like electric convection: it looks as if the electric energy were carried
across the terminals by luminous material particles, which are shot off, in straight lines, from the negative terminal with great energy, producing luminosity where they strike; and after losing their luminosity have to find their way to the positive terminal. The transfer of electric energy by the cathode ray would then have the same relation to the transfer of electric energy by a copper wire as the transfer of kerosene by a series of tank.

Assuming then the hypothesis that the cathode ray is the transfer of electric energy by convection by material particles, we will see what conclusions we can derive therefrom.

A material particle containing electric energy is acted upon by an electrostatic field, in a direction depending on the polarity of the electric energy, whether positive or negative against surrounding space. The would be deflected by an electrostatic field by an amoun depending on the intensity of the field and on the energy, mass and velocity of the material particles. This is the case: the cathode ray is deflected, and measure-
ments of the deflection of this ray by the electrostatic field thus give us a relation between electric energy, mass and velocity of the cathode ray particles. Moving electric energy, whether flowing through a metal conductor or carried by a moving particle, is acted upon by a by a magnetic field by anamount depending on the electric energy, mass and velocity of the moving cathode particles. This is the case. From these two relations, given by the deffection of the cathode ray by the electrocalculate by the magnetic field, respectively, we can cathode ray particles. If the masses of the cathode ray particles, calculated by this assumption, were found to be of the same magnitude as masses of other particles, calculated by other means, such as the chemical atoms or molecules, if their velocities were comparable with other known velocities, this would be a strong confirmation of the hypothesis of electric convection by the eathode ray, that is, of the ionic theory. However, this is not the case, and the experiment therefore neither confirms nor contradicts the ionic theory. The calculation shows that, if the conduction of the vacuum tube is by convection of electric energy by moving particles, these particles, called electrons, must be very much smaller than the chemical atoms, or of a magnitude of one-thousandth the size of the smallest chemical atom, the hydrogen atom. Their velocity of motion must be inconceivably high--comparable with, though smaller than the velocity of light. They carry electric energy at a negative potential against surrounding space, that is, the electron may be considered as the negative terminator of a line of dielectric force, while the positive end of this line of dielectric force terminates at the positive terminal of the vacuum tube, or at a positive electron, where such exists.

The question then arises: What is the electron? By the derivation of its hypothetical existence, it is the action of forces on its mechanical momentum. It thus would be a new form of matter, a new chemical atom, a thousand times smaller than the hydrogen atom. It has been called "an atom of electricity." As "electricity" is a vague term without physical meaning, which has loosely been used for "electric quantity" (and even "electric quantity" is a mere mathematical fiction, a component factor of electrical energy) no objection exists to giving the name "electricity" by the electron. It naturally does not explain anything: The electron certainly is not electric quantity, thing: The electron eertainly is not electric quantity, form of matter which is the carrier of electric energy. Then, however, the electron in its definition comes rather close to the hypothetical ether atom, which is the carrier of radiant energy, that is, the carrier of the energy of the electric wave in space.

The electron, however, can not be considered as electric energy, nor as representing or carrying a definite amount of electric energy, even when assnoisteit with a definite quantity of electricity, no more than the iron atom of a magnetic circuit can be considered as magnetic energy, or as carrier of a definite amount of magnetic energy. Energy comprises the product of quantity and intensity, and the electric energy carried by the electron is its electric quantity times the intensity of its electric field, that is, the potential gradient along up to a reference point on this line of dielectric forcethe potential of the positive terminal, of surrounding space, of the universe, or anything else. This disposes of the mistaken conception, occasionally expressed, that the electron represents a definite amount of electric energy, and leaves the amount of energy of the electron indefinite, that is, depending on an arbitrarily chosen reference potential, just as it is with any other form such as a moving body, is always relative, depending on a reference point.

Obviously, then, electric energy can not be measured by the number of electrons, and has no direct relation to it, but depends on the electric intensity, or potential difference.

In the last years, the ionic theory has been greatly strengthened by the discovery and investigation of phenomena similar to those of the cathode ray, though more general in nature, in the radiation of so-called
"radio-active substances." A number of chemical elements, such as radium, thorium, uranium, etc., continuously send out rays of various kinds. Some., continuously send out rays of various kinds. Some
of these, the $\beta$ rays, are identical with the cathode rays of the vacuum tube, or, in other words, are deflected in the same manner by electrostatic and magnetic fields, and are therefore considered as electronsterminators of the negative end of a line of dielectric force, of a mass about a thousandth that of the hydrogen
atom, shot off by the radio-active substance with veloeities approaching that of light. Other rays, the a rays, are deflected in an opposite direction by electrostatic and magnetic fields, and thus must be considered as carriers of electric energy of positive potential: positive electrons. Their mass, as calculated in the manner above described, is that of the helium atom (4 times the mass of the hydrogen atom), and they are therefore generally considered as helium atoms carrying electric energy of positive potential. They velocities of the negative electron, though still incon- 
reivably high. When carrying electric energy, they rontain the same quantity of electricity at positive potential that the negative electrons carry at negative potential, and if the latter are considered as terminators of the negative end of a line of dielectric force, the helium atoms as positive electrons are terminators of the positive end of a line of dielectric force.

A third class of rays, issuing from radio-active substances, are the $\gamma$ rays. They have the same characteristies as the other rays, except that they are not deflected by electrostatic or magnetic fields. They are identical in their properties with the X-rays, discussed above as electric waves at the extreme end cussed above as electric waves at the extreme end
of high frequencies, and are usually considered as $\mathrm{X}$-rays.

Here we come to one of those conclusions which do not appear rational: the $\alpha, \beta$ and $\gamma$ rays are very similar in their nature, differing only by the direction and amount of their deflection, and it therefore does not appear reasonable to assume that the $y$ rays are ether appear reasonable to assume that the $\gamma$ rays are ether
waves, while the $\alpha$ and $\beta$ rays are projectiles thrown waves, while the $\alpha$ and $\beta$ rays are projectiles thrown
off by the radio-active mass. The attempt of avoiding off by the radio-active mass. The attempt of avoiding
this dilemma by assuming the $\gamma$ rays to be projectiles, this dilemma by assuming the $\gamma$ rays to be projectiles,
which carry equal positive and negative electric quantity, which carry equal positive and negative electric quantity,
and therefore are not deflected, appears forced and merely transfers the difficulty into the relation between $\mathrm{X}$-rays and ultra-violet light. The latter is generally conceded-and corroborated by the phenomena of interference, etc.- to be ether waves. At the extreme ultra-violet, however, the properties begin to shade ultra-violet, however, the properties begin to shade
into those of the X-rays, and it again appears unreainto those of the X-rays, and it again appears unrea-
sonable to assume such an essential difference between sonable to assume such an essential difference between
ultra-violet and X-ravs, as that the ones are ether waves, ultra-violet and X-rays,
the others projectiles.

In many instances, when we follow the reasoning of the ionic theory to its conclusion, we meet contradictions. For instance, the calculation of the mass of the electron shows that at very high velocities the mass is not constant, but increases with increasing velocity, becoming infinity; and therefore the kinetic energy of becoming infinity; and therefore the kinetic energy of the electron becomes infinite, if its velocity equals the
velocity of light. This is impossible, as it contradicts the law of conservation of energy: if we consider two electrons, moving in opposite directions at half the velocity of light, their kinetic energy against surrounding space would be finite. Their relative motion against each other, however, is at the velocity of light, and their kinetic energy against each other would be infinite. Since, however, they were set in motion by finite energy, their relative energy can not be infinite. To overcome this difficulty, a fictitious or apparent mass-the "electromagnetic mass"- has been attributed u. th electrons, which is not the mass of mechanies. However, the cis'sulation of mass and velocity of the electrons is based on the kinetic energy of the electron, that is, on its mechanical mass, and not a new kind of mass, which is not a mass in the mechanical sense. These and other numerous contradictions to which the conception of the ionic theory leads, obviously the conception of the ionic theory leads, obviously
do not mean that the ionic theory is fundamentally do not mean that the ionic theory is fundamentally
wrong in principle: we have also seen that the wave wrong in principle: we have also seen that the wave
theory of radiation, in the properties of the luminiferous ether, lead to attributes that are contradictory and thereby impossible. We find the same thing in all theoriesthe chemical, the thermodynamic, etc. It simply means that our present formulation of the ionic theory, of the electromagnetic wave theory, and of all other
theories are very far from final correctness, but are at theories are very far from final correctness, but are at
best only very crude conceptions of the nature of things, best only very crude conceptions of the nature of things,
which will have to be modified again and again with our increasing knowledge before we can expect to reach a moderately rational conception of nature's laws and phenomena, if we ever arrive there.

\section{The Magnetic Field of Cathode Rays}

ONE characteristic property of the eathode rays is the fact that they are deflected by the magnet. These rays, as is well known, consist of electrons projected into a vacuum tube (Fig. 1) from an electrode charged to a high potential, and behave exactly like an ordinary conductor carrying a current. The question naturally arises whether such a cathode ray produces a magnetic field of its own, corresponding in character to that which an equivalent conductor would create, as indicated in Fig. 2. Although there was every probahility that this should be the case, numerous experiments devised to furnish positive proof of the fact failed to

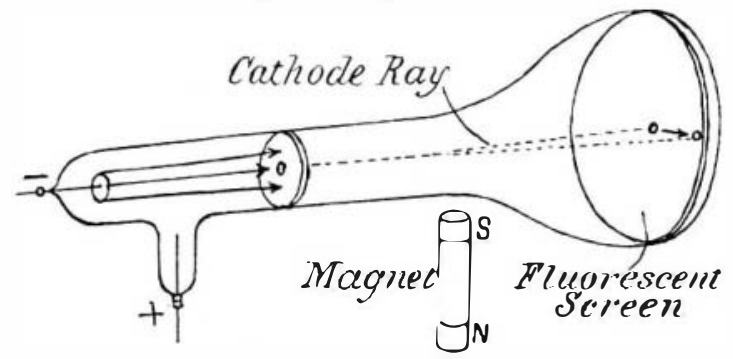

Fig. 1.-The Magnet Deflects the Cathode Ray in the Direction of the Arrow Seen on the Fluorescent Screen.

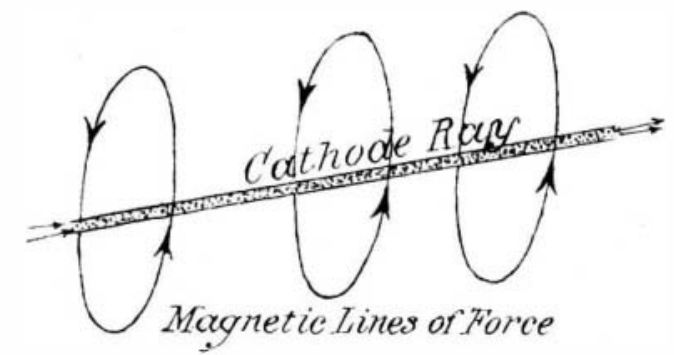

riy. 2.-Diagram showing the Relation of the Magnetic Lines of Force to the Cathode Rily.

give the desired result. On this account special interest is attached to a recently published work of Joffe, noted in Prometheus, which furnishes the required evidence. This observer made the cathode ray part of a closed circuit, The current represented by the cathode ray could be measured by means of a galvanometer $G$ (Fig.
3 ). It was then found that within the close limits of

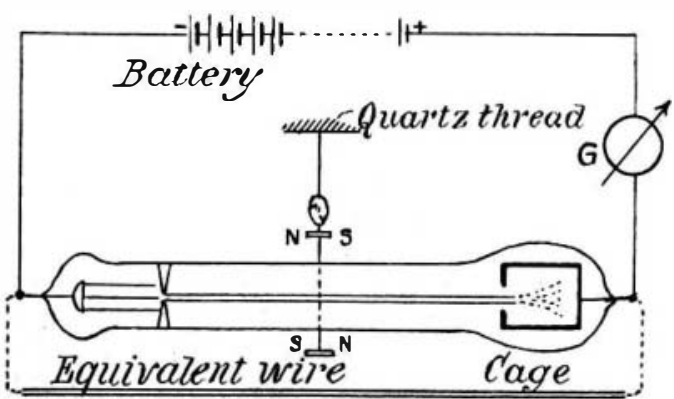

Fig. 3.-Diagram of Toffe's Experimentill Arrange-

experimental error the same deflection was caused in a small pair of magnets suspended from a quartz thread (see Fig. 3) whether the current was transmitted in the form of a cathode ray or through an equivalent copper wire inserted as nearly as possible at the same place. The relation between the deflection of the magnet and the strength of the current was the same in both cases

\section{A Simple Method of Preparing Pure} Samples of the Rare Gases

The methods hitherto described for the preparation of pure specimens of the rare gases labor under the severe disadvantage of being extremely tedious and time-consuming, and do not even always yield a satisfactory result at that. Especially the complete elimination of hydrogen and carbon-monoxide is attended with th greatest difficulties. A process described by Gebllhoff i the proceedings of the German Physical Society, 1911. and presented in brief extract in Prometheus, from which our illustration is taken, therefore deserves specia notice. The principle of the process depends on the fact

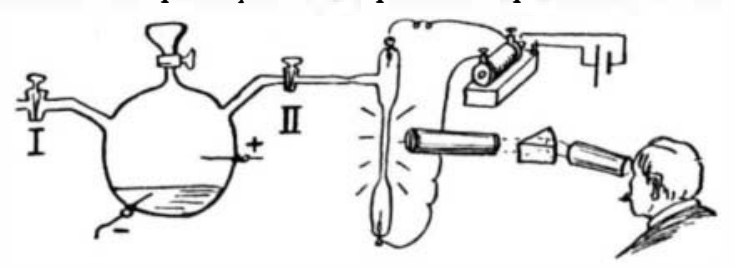

Ise of lotassium in the Ireparation of the Ratre Giases

that vapors of the alkali metals will, under the influence of the silent electric discharge, so completely absorb hydrogen, carbon-monoxide, oxygen, and nitrogen, that these gases can no longer be detected by the spentroscope. Gehlhoff uses a vapor of the comparatively cheap metal potassium, which acts very energetically at 200 degrees. The apparatus adapted for this purpose i shown in our illustration. The purif ying flask is connected through tap $I$ to the air-pump, a charge of potassium being introduced through a separate opening. The flask is then filled with the impure rare gas. B tween the potassium, which serves as cathode, and an anode fused in through the wall of the flask, a silent discharge is established. After but a few minutes the gas is pure, and a Geissler tube fed through tap $I I$ shows to the observer through the spectroseope the pure spectrum of the gas under treatment.

\section{Limiting Value of the Shortest Possible Sound Waves}

IT is well known to every student in elementary physies that sound is propagated in the form of waves of alternate compression and dilatation. In air its velocity is about 1,100 feet per second. The frequency of the waves varies according to the nature of the source, and is recognized by the ear, the sound heard being the "higher" in pitch the greater the frequeney, or, what amounts to the same thing, the smaller the wave-length. Thus, for example, if on striking a bell (Fig. 2), 220 vihrations per second are produced, the length $l$ of one wave, or in other words, the distance, measured in the direction of propagation, between two consecutive points in the air at which conditions are precisely similar will be given by

$$
\frac{1.100}{220}=5 \text { feet. }
$$

A simple means for determining the length of sound waves is furnished by the so-called Kundt's dust tube. This is represented in Fig. 2. A glass rod firmly clamped at its middle in a suitable support, as shown on the

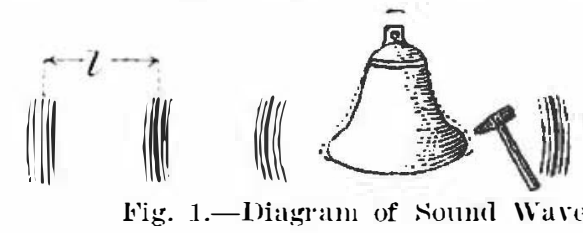

right-hand side of the drawing, is set vibrating by rubbing it with a piece of wet leather held in the hand. The farther end of the rod, which carries a cardboard disk, extends into the dust tube, the air in which is set vibrating. By adjusting a piston inserted at the opposite end of the tube, the length of the vibrating air colum can be regulated until it is an exact multiple of the wavelength of the sound produced by the rod. The instant when this occurs is easily recognized if the tube has been sprinkled with some fine powder, such as lycopodium. For as soon as the right adjustment is reached this powder arranges itself in little clusters or heaps at regular intervals, corresponding to the distance between the

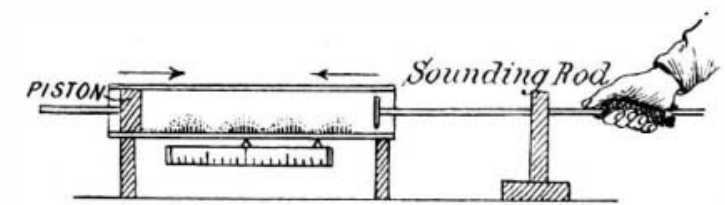

Fig. 2.-Kinndt's I)ust Tule.

crests of the sound waves. This distance can then be measured by means of a suitable scale, as indicated in the drawing.

The Russian physicist Lebedew has recently established the fact that, while sound waves of a medium wave-length suffer very little absorption in the course of their propagation, this absorption becomes very considerable in the case of short waves. The following table gives the distance $D$ in centimeters from the sounding source at which a wave of wave length $l$ is reduced to one-hundredth of ats original intensity:

$\begin{array}{cc}\text { Wave-length ، } & \text { Distance } D \text { from the Source } \\ \text { Millimeters. } & \text { Centimeters. } \\ 0.8 & 40 \\ 0.4 & 10 \\ 0.2 & 2.5 \\ 0.1 & 4.6\end{array}$

From this table it will be seen that 110 of a millimeter represents about the limit. of the length of sound waves accessible to observation.-Prometheus.

\section{Artificial Production of Well Water}

THE only water suited for city supply systems is mineralized well water, containing nitrogen only in the highest degree of oxidation. As, however, this underground water mostly fails to suffice for actual requirements, at tempts have been made to mix it with river water, imparting to the latter by natural filtration all the properties of underground water.

At a recent meeting of the Geological Association, at Frankfort-on-the-Main, Baurat Scheelhaase gave an interesting account of his method for converting river water into well-water, which has already been carried out into practice with satisfactory results. The water of the river Main, having passed through artificial filters, i allowed to ooze thorugh the soil. After having reached a certain depth in about three weeks, this water filter through a distance of 500 meters as far as the Oberforsthaus pumping station. Even at 425 feet distance from its entrance into the soil, the Main water is found to be equivalent to the natural underground water. It be equivalent to the natural underground water. It
takes three years to arrive from the point of entrance to the pumping station. The output of the plant is 14,120 cubic feet of water per day.

\section{TABLE OF CONTENTS}

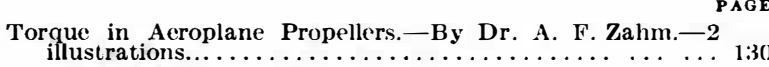
The Purity of Natural Waters.-I. By Maximilian Marsson 131

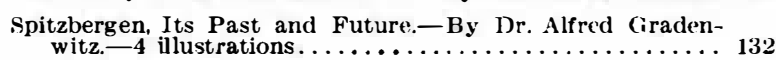

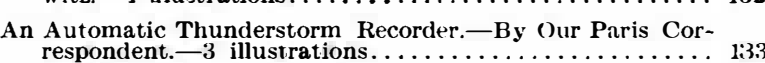

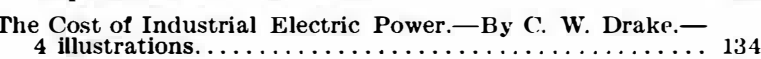
A Scientific Study of Seasickness.................. 135

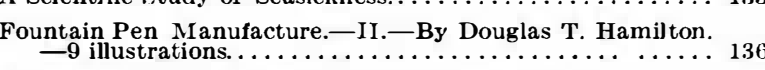
Phosphoric Acid and the Quality of French Wines........ 137 The Problem of the Railway Rail.-By Rohert G. Skerrett. Fuel Consumption and the Climate of Cities............ 139

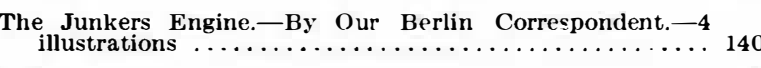
Japanese Fans...

Electric Disturbances and the Nature of Electrical Energy. The Magnetic Field of Cathode Rays................. 144 A Simple Method of Preparing Pure Samples of the Rare Limiting the Value of the Shortest Possible Sound Waves... 144 Artiflcial Production of Well Water.................. 144 